\title{
Numerical study of multiple bubble dynamics with the BEM-ACA approach
}

\author{
Z. Fu \& V. Popov \\ Wessex Institute of Technology, Environmental and Fluid Mechanics, \\ Southampton, U.K
}

\begin{abstract}
Multiple bubble dynamics is analysed involving interactions between bubbles and also between bubbles and adjacent boundaries. In the current work, the adaptive cross approximation (ACA) BEM model is employed to study a "bubble cloud". Three major factors are discussed, i.e., the presence of an adjacent solid wall and bubble volume fraction. The impact of the cloud on surrounding boundaries is studied.
\end{abstract}

Keywords: multiple bubble dynamics, bubble cloud, adaptive cross approximation, boundary element method.

\section{Introduction}

The numerical study of a number of bubbles has been documented before. Chahine performed a simulation with 21 bubbles using a BEM code called 3DynaFS [1]. By using a fast approach i.e. FFTM clustering, Bui et al. [2] modelled the evolution of up to 25 bubbles in a plane. The current authors also developed a fast approach for bubble dynamics based on the adaptive cross approximation (ACA) approach, leading to the ACA/BEM model [3]. However, the physics of multiple bubble dynamics has not been well studied due to the prohibitively expensive computational cost of the relatively large-scale problems and manifested randomness of evolution of each individual bubble.

In the current work, we studied the physical behaviour of multiple bubbles under the excitation of ultrasound. Three factors including the presence of a nearby wall and bubble volume fraction are discussed. Potential contour and the distribution of kinetic energy density are introduced to help analyze the dynamics of liquid flow within the cloud. The numerical simulations are based 
on the ACA/BEM model. Results of modelling including up to one-hundred bubbles are presented.

\section{Theoretical formulations for the ACA approach}

Bubbles are assumed to be in a potential flow, which satisfies the Laplace equation, i.e. $\Delta \phi=0$ (where $\phi$ is the velocity potential). The harmonic function $\phi$ can be represented by a potential distribution [4]. Applying the single-layer potential yields:

$$
\phi(x)=\int_{\partial \Omega} \sigma(\xi) \mathrm{G}(x, \xi) \mathrm{ds}(\xi),
$$

where $\partial \Omega$ is the manifold of the flow domain. $x$ and $\xi$ are a source point and an integration point, respectively. In the $\mathbb{R}^{3}$ space, the fundamental solution of the Laplace equation $\mathrm{G}(x, \xi)$ takes the form:

$$
\mathrm{G}(x, \xi)=1 /|x-\xi| .
$$

The system matrix generated by eqn. (1) can be partitioned into a number of admissible submatrices. Then, the ACA algorithm is applied to produce a representation of each $m \times n$ admissible submatrix efficiently, leading to $\mathrm{O}\left((\mathrm{m}+\mathrm{n}) \mathrm{r}^{2}\right)$ operations and $\mathrm{O}((\mathrm{m}+\mathrm{n}) \mathrm{r})$ memory consumption (where $\mathrm{r}$ is the rank $<<\operatorname{Min}(\mathrm{m}, \mathrm{n}))$. The reader is referred to [3] for the details of the ACA approach in bubble dynamics. In this work, the ACA algorithm [3] is extended and its performance improved.

Eqn. (1) implies that the collocation scheme is employed for the assembly of the algebraic system. Surface integration might dominate the assembly process, taking the majority of the required CPU time [5]. The direct application of Algorithm 1 for an $m \times n$ submatrix $\mathcal{M}$ from the system matrix, i.e. $\mathcal{K}=\mathcal{M}$, requires computation of $\mathrm{O}((\mathrm{m}+\mathrm{n}) \mathrm{r})$ surface integrations.

Algorithm 1: The ACA algorithm [3].

Let $\mathcal{K}$ be the $\mathrm{m} \times \mathrm{n}$ admissible matrix in accordance with the pair of source and integration node sets $(\mathrm{X}, \Xi)$, and $\mathcal{R}$ be of the same size as $\mathcal{K} . \mathcal{J}$ is the identity matrix and $\mathcal{J}_{\mathrm{k}}$ is denoted as the $\mathrm{k}$-th column vector of $\mathcal{J}$. $\mathcal{R}=\mathcal{K}, \mathrm{i}_{1}=1$. For $\mathrm{k}=0,1$, $\underline{2, \ldots, \text { compute }}$

$$
\begin{aligned}
& \mathcal{J}_{\mathrm{i}_{\mathrm{k}+1}^{\mathrm{T}}}^{\mathrm{T}} \mathcal{R}=\mathcal{J}_{\mathrm{i}_{\mathrm{k}+1}^{\mathrm{T}}} \mathcal{K}-\mathcal{U}_{\mathrm{i}_{\mathrm{k}+1,1 \mathrm{k}}} \mathcal{V}_{1: \mathrm{n}, 1: \mathrm{k}}^{\mathrm{T}} \\
& \operatorname{Max}\left\{\left|\mathcal{R}_{\mathrm{i}_{\mathrm{k}+1, \mathrm{j}}}\right|\right\}(\neq 0) \rightarrow \mathrm{j}_{\mathrm{k}+1} \text {; } \\
& \mathcal{V}_{1: \mathrm{n}, \mathrm{k}+1}^{\mathrm{T}}=\mathcal{J}_{\mathrm{i}_{\mathrm{k}+1}^{\mathrm{T}}}^{\mathrm{R}} / \mathcal{R}_{\mathrm{i}_{\mathrm{k}+1}, \mathrm{j}_{\mathrm{k}+1}} ; \\
& \mathcal{U}_{1: \mathrm{m}, \mathrm{k}+1}=\mathcal{K} \mathcal{J}_{\mathrm{j}_{\mathrm{k}+1}}-\mathcal{U}_{1: \mathrm{m}, 1: \mathrm{k}} \mathcal{v}_{\mathrm{j}_{\mathrm{k}+1}^{\mathrm{T}}, 1: \mathrm{k}} \\
& \mathrm{c}_{\text {stop }}=\varepsilon_{\mathrm{a}}\left\|\mathcal{U}_{1: \mathrm{m}, 1: \mathrm{k}} \mathcal{V}_{1: \mathrm{n}, 1 \mathrm{k}}^{\mathrm{T}}\right\|_{\mathrm{F}}-\left\|\mathcal{U}_{1: \mathrm{m}, \mathrm{k}+1}\right\|_{\mathrm{F}}\left\|\mathcal{V}_{1: \mathrm{n}, \mathrm{k}+1}\right\|_{\mathrm{F}},\left(\mathrm{c}_{\text {stop }}>0, S T O P\right) \text {; } \\
& \operatorname{Max}\left\{\left|\mathcal{U}_{\mathrm{i} \neq \mathrm{i}_{\mathrm{k}+1}, \mathrm{k}+1}\right|\right\}(\neq 0) \rightarrow \mathrm{i}_{\mathrm{k}+2} \text {. }
\end{aligned}
$$

( $\varepsilon_{\mathrm{a}}$ is the prescribed precision for the algorithm.) 
Alternatively, Algorithm 1 is applied for $\mathcal{K}=\mathrm{G}(\mathrm{X}, \Xi)$ instead of the defined submatrix $\mathcal{M}$ above. Surface integrations are only required in the chosen $\mathrm{r}$ rows. The modified ACA algorithm (i.e. ACA+) is presented in Algorithm 2, which demonstrates $\mathrm{O}(\mathrm{nr})$ surface integrations, compared with $\mathrm{O}((\mathrm{m}+\mathrm{n}) \mathrm{r})$ when only the ACA algorithm is applied.

Algorithm 2: The ACA+ algorithm.

Let $\mathcal{M}$ be an $\mathrm{m} \times \mathrm{n}$ admissible block from the system matrix with the source node set $X$ and the integration node set $\Xi(\mathrm{m}=\# \mathrm{X}, \mathrm{n}=\# \Xi)$, and $\mathcal{K}=\mathrm{G}(\mathrm{X}, \Xi)$.

Conduct the ACA algorithm for $\mathcal{K}$ and record row indices $i_{1: r}$ and column indices $\mathrm{j}_{1: \mathrm{r}}$;

$$
\begin{gathered}
\mathcal{K}_{1: \mathrm{m}, 1 \mathrm{r}}^{\prime}:=\mathcal{K}_{1: \mathrm{m}, \mathrm{j}_{1: \mathrm{r}}} ; \\
\mathcal{W}_{1: \mathrm{r}, \mathrm{r}}:=\mathcal{K}_{\mathrm{i}_{1: \mathrm{r}} \mathrm{j}_{1: \mathrm{r}}} ; \\
\mathcal{M}_{1: \mathrm{r}, \mathrm{r}: \mathrm{n}}^{\prime}:=\mathcal{M}_{\mathrm{i}_{1: \mathrm{r}}, \mathrm{n}, \mathrm{n}} ; \\
\mathcal{M} \approx \mathcal{K}^{\prime} \mathcal{W}^{-1} \mathcal{M}^{\prime}=\mathcal{C} \mathcal{M}^{\prime}, \text { with } \mathcal{C}:=\mathcal{K}^{\prime} \mathcal{W}^{-1} .
\end{gathered}
$$

\section{Results and analysis}

We apply the ACA/BEM model to simulate the evolution of the group of bubbles, denoted as "bubble cloud", and study the dynamics of liquid within the cloud.

Our simulations start with the generation of a number of bubbles that are populated randomly in a cube filled with ethanol. The parameter "bubble volume fraction", i.e. $\beta$, is introduced as the ratio between the aggregate volume of bubbles and the cubic volume $\mathrm{V}_{\mathrm{c}}$ :

$$
\beta=\sum_{\mathrm{i}}^{\mathrm{n}_{\mathrm{b}}} V_{\mathrm{b}, \mathrm{i}} / \mathrm{V}_{\mathrm{c}},
$$

which indicates the population density of bubbles within the liquid. The characteristics of a bubble cloud can be analysed using two parameters: i.e. distributions of velocity potential $\phi$ and kinetic energy density $E_{k}$. The density of kinetic energy $\mathrm{E}_{\mathrm{k}}$ is defined as:

$$
\mathrm{E}_{\mathrm{k}, \mathrm{d}}=\frac{1}{2} \rho_{\mathrm{L}}|\overrightarrow{\mathrm{v}}|^{2} .
$$

Higher $\mathrm{E}_{\mathrm{k}, \mathrm{d}}$ suggests higher rate of liquid flow at the corresponding location.

\subsection{The presence of a solid wall}

The presence of a solid boundary near a bubble cloud leads to a half-space problem. When the cloud is close enough, the evolution of bubbles might be affected by the wall. Figure 1 presents a group of twenty-five bubbles along a plane $\mathrm{z}=0$. These bubbles of a uniform initial size $\mathrm{R}_{0}=10 \mu \mathrm{m}$ are randomly distributed with bubble volume fraction $\beta=1.0 \%$. The bubble evolution is driven by an acoustic wave of $f=20 \mathrm{kHz}$. Physical parameters used in the simulation of the bubble evolution are presented in Table 1 . 
Table 1: Physical parameters used for the study of multiple bubble dynamics.

\begin{tabular}{llll}
\hline Initial vapour pressure $\mathrm{p}_{\mathrm{v} 0}(\mathrm{~Pa})$ & $29,356.9$ & Liquid density $\rho_{\mathrm{L}}\left(\mathrm{kg} \cdot \mathrm{m}^{-3}\right)$ & 766 \\
Initial gas pressure $\mathrm{p}_{\mathrm{g} 0}(\mathrm{~Pa})$ & $71,968.1$ & Initial velocity potential $\phi_{0} 0$ \\
Gas ratio of specific heat $\gamma_{\mathrm{g}}$ & 1.4 & Wave frequency $f(\mathrm{kHz})$ & 20 \\
Vapour ratio of specific heat $\gamma_{\mathrm{v}} 1.13$ & Wave amplitude $\mathrm{p}_{\mathrm{amp}}(\mathrm{MPa}) 1.0$ \\
Liquid temperature $\mathrm{T}_{\mathrm{a}}\left({ }^{\circ} \mathrm{C}\right)$ & 50 & Wave phase $\theta$ & $\pi / 2$ \\
Initial bubble size $\mathrm{R}_{0}(\mu \mathrm{m})$ & 10 & Wave source location $x_{\mathrm{s}}(\mathrm{m})\left(10^{-3}, 0,0\right)$ \\
\hline
\end{tabular}

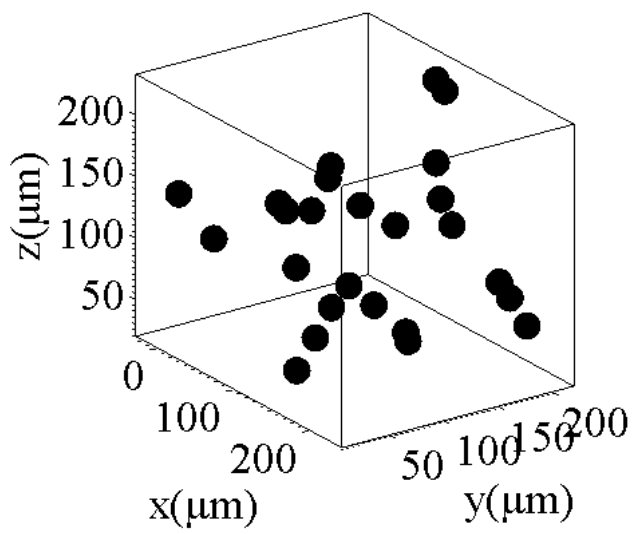

Figure 1: $\quad$ Twenty-five randomly distributed bubbles, each with $\mathrm{R}_{0}=10 \mu \mathrm{m}$ in a volume of liquid $(\beta=1.0 \%)$.

The wave of $f=20 \mathrm{kHz}$ proves to be a subresonant wave condition since an immediate collapse of some bubbles is seen at the end of simulation $\mathrm{t}=0.6086 \mu \mathrm{s}$. Figure 2 presents the results at the instant of $\mathrm{t}=0.6086 \mu \mathrm{s}$. The differences in distributions of potential and kinetic energy density are comparable when the bubble cloud is in an open field and when it is adjacent to a solid wall (i.e. $z=0$ ).

Velocity potential distribution can help to identify the interactions between bubbles. The negative values of potential for both cases in Figure 2(a) and (b) imply a general process of bubble size decrease at the instant. Evident potential gradients can be observed in liquid close to bubbles. In (a), areas on the side of the cloud are hardly affected by the evolving bubbles as approximately "zero" potential can be seen. By contrast, the dominant higher absolute values of potential in (b) suggest that a larger volume of liquid is influenced by the evolving bubbles. With respect to kinetic energy density $E_{k}$, less evident difference between Figure 2(c) and (d) can be seen. High values in a long and narrow area of the wall surface demonstrate notable impacts of the solid wall when the liquid is close (see Figure 2(d)). 


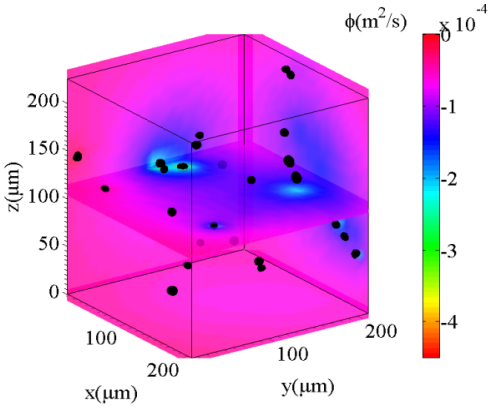

(a)

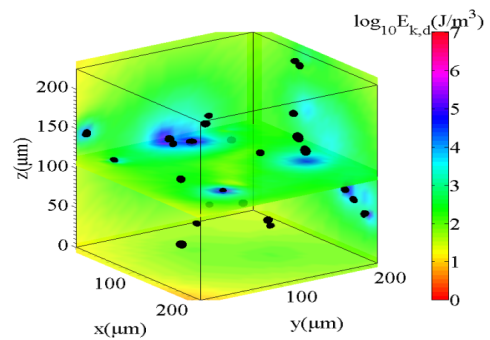

(c)

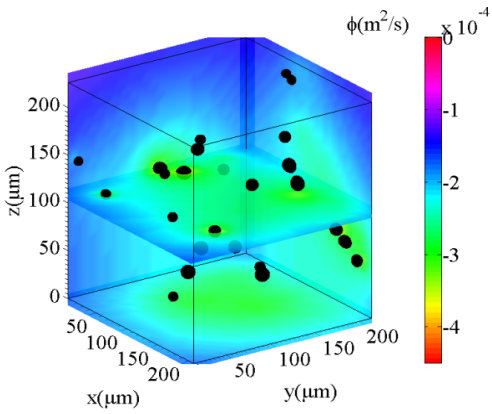

(b)

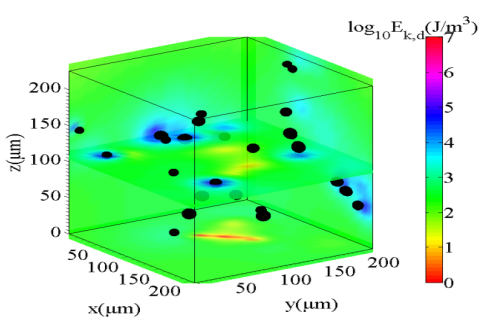

(d)

Figure 2: Distributions of $\phi$ and $E_{k, d}$ in a bubble cloud of 25 bubbles at $\mathrm{t}=0.6086 \mu \mathrm{s}$. (a) $\phi$ (no wall); (b) $\phi$ (near a wall); (c) $\mathrm{E}_{\mathrm{k}, \mathrm{d}}$ (no wall); (d) $E_{k, d}$ (near a wall).

The kinetic energy and the Kelvin impulse are presented in Figure 3. The kinetic energy $E_{k}$ experiences an increase from zero, owing to the excitation by the acoustic wave. Towards the end of the bubble collapse, the increase in the internal pressures slows down the evolution process, thereby reducing $\mathrm{E}_{\mathrm{k}}$. The variation of $E_{k}$ also shows a slow-downward process with the presence of the solid boundary. With respect to the Kelvin impulse, the most evident difference is observed in the z-direction. The presence of the boundary leads to higher impulse towards the wall. By comparing the scale of the Kelvin impulse it is found that the differences in the Kelvin impulse in the $\mathrm{x}$ - and $\mathrm{y}$-directions are negligible.

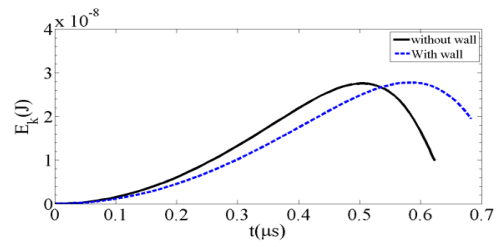

(a)

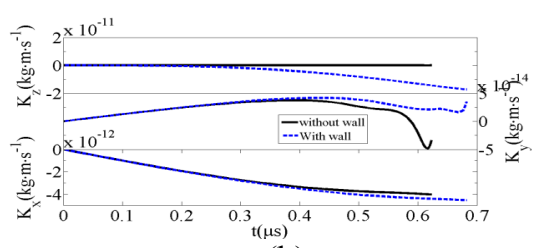

(b)

Figure 3: Variations of the kinetic energy and Kelvin impulse with and without the presence of a solid wall. (a) $\mathrm{E}_{\mathrm{k}}$; (b) $\mathrm{K}_{\mathrm{x}}, \mathrm{K}_{\mathrm{y}}$ and $\mathrm{K}_{\mathrm{z}}$. 


\subsection{Bubble volume fraction}

The volume fraction of bubbles in liquid $\beta$ specifies the average distance between bubbles when the number of bubbles is fixed - the higher $\beta$ corresponds to the denser population of bubbles within a cloud. Bubbles with sufficiently high $\beta$ yield strong interactions between each other, owing to the close distances.

To study the impacts of bubble volume fraction on the dynamics of a bubble cloud, we introduce an example of 50 randomly distributed bubbles in a volume of liquid. Figure 4 (a) and (b) illustrate the bubble clouds for the case of $\beta=1.0 \%$ and $5.0 \%$, respectively. A solid boundary is present at the bottom at a distance of $20 \mu \mathrm{m}$ from the nearest boundary of the liquid volume. The same physical parameters as in are used in the simulations.

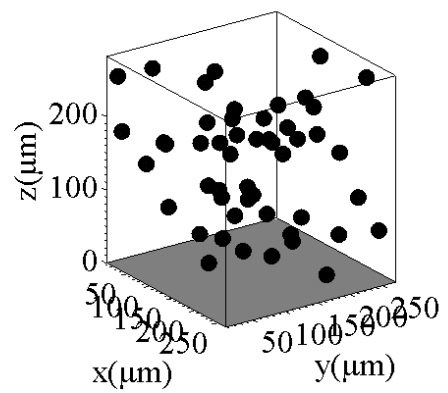

(a)

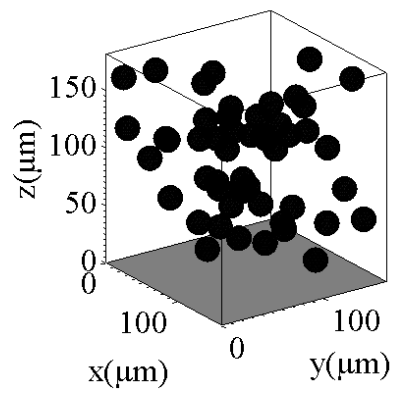

(b)

Figure 4: Fifty bubbles of $\mathrm{R}_{0}=10 \mu \mathrm{m}$ randomly distributed in a volume of liquid with a solid wall at $\mathrm{z}=0 \mu \mathrm{m}$. (a) $\beta=1.0 \%$; (b) $\beta=5.0 \%$.

Bubbles experience collapse under the prescribed wave condition and the distributions of the potential and kinetic energy at $t=0.7825 \mu$ s are presented in Figure 5. Both cases show significant variations in the potential on the side of the cloud compared to the areas in the middle. The liquid is expected to be flowing into the cloud's area as the potential increases with the distance from the cloud centre. The larger range of potential values, i.e. $-5 \times 10^{-4}--2.5 \times 10^{-4} \mathrm{~m}^{2} / \mathrm{s}$ can be observed for the case of $\beta=5.0 \%$, which indicates higher interactions of bubbles.

The contours of $E_{k, d}$ in Figure 5 (c) and (d) show high concentration of kinetic energy in liquid areas surrounding the bubbles. On the side of bubble cloud, the predominant $E_{k, d}$ of $O\left(10^{3.5}\right)$ for the case of $\beta=5.0 \%$ versus $O\left(10^{2.5}\right)$ for the case $\beta=1.0 \%$ implies faster liquid flow towards the cloud when bubble volume fraction increases. Due to the limited gap in the bubble cloud, liquid with high bubble volume fraction may show slower velocity, as is seen in Figure 5 (d) of low $\mathrm{E}_{\mathrm{k}, \mathrm{d}}$ in some areas of the middle plane. Figure 6 presents the velocity contour on the surface of the solid wall. Flowing liquid with the velocity range of 0.10 $2.33 \mathrm{~m} / \mathrm{s}$, for the case of $\beta=5.0 \%$, versus $0.02-1.92 \mathrm{~m} / \mathrm{s}$, for the case of $\beta=1.0 \%$, demonstrates enhanced impacts on the wall when bubbles are populated with higher volume fraction. 


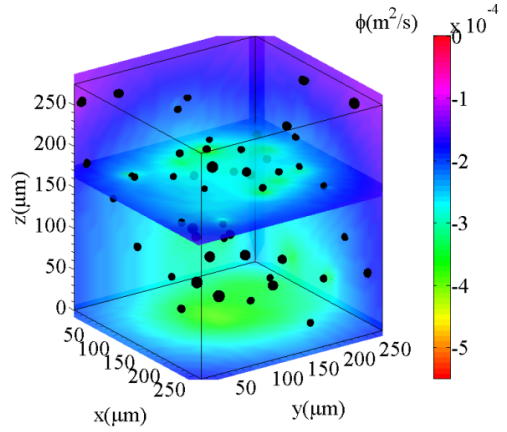

(a)

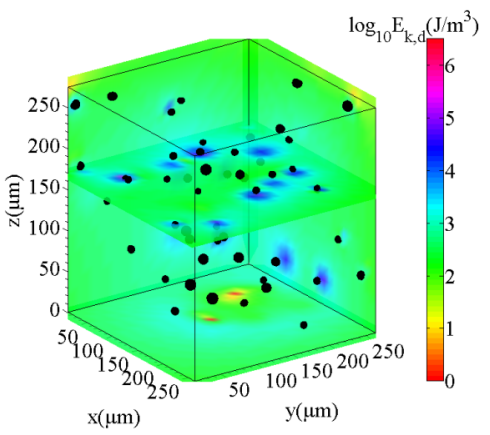

(c)

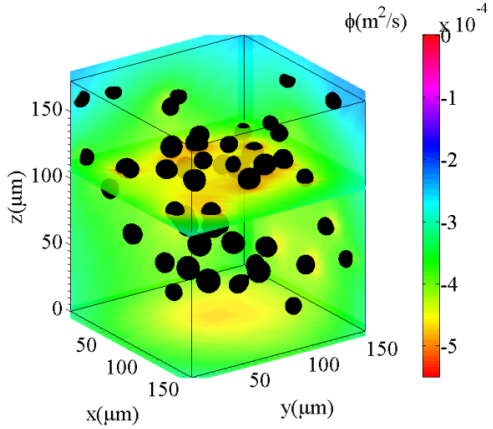

(b)

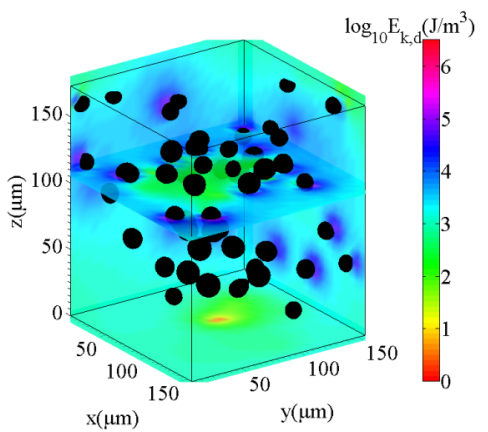

(d)

Figure 5: Distributions of $\phi$ and $E_{k, d}$ in bubble clouds of different $\beta$ ( $t=0.7825$ $\mu \mathrm{s})$. (a) $\phi(\beta=1.0 \%)$; (b) $\phi(\beta=5.0 \%)$; (c) $\mathrm{E}_{\mathrm{k}, \mathrm{d}}(\beta=1.0 \%)$; (d) $\mathrm{E}_{\mathrm{k}, \mathrm{d}}$ $(\beta=5.0 \%)$.

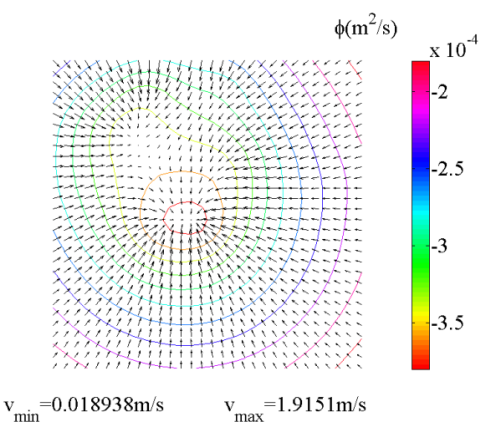

(a)

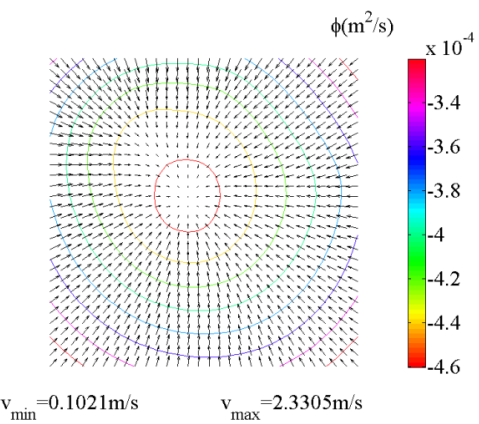

(b)

Figure 6: Velocity distributions in bubble clouds of different $\beta(\mathrm{t}=0.7825 \mu \mathrm{s})$. (a) $\beta=1.0 \%$; (b) $\beta=5.0 \%$. 


\section{Conclusions}

Numerical modelling of multiple bubble dynamics has been carried out. The impacts of a cloud of bubbles on surrounding liquid have been discussed by analysing the distributions of potential and density of kinetic energy. The presence of a solid boundary appears to improve interactions within a bubble cloud which is manifested in a higher variation of potential. Also, the more evident density of kinetic energy indicates that liquid flow on the surface of the solid boundary is significantly accelerated. The bubble volume fraction can be used to indicate the strengths of interactions between bubbles and impacts on the adjacent boundary. Owing to relatively closer distances with higher volume fraction, bubbles within the cloud have stronger influences on each other and a higher speed of liquid flow can be seen on the boundary surface.

\section{Acknowledgement}

The present study was supported by the SONO project, contract number: 228730, as part of the Seventh Framework Programme (FP7-NMP-2008-Large2).

\section{References}

[1] G.L. Chahine, "Strong interactions bubble/bubble and bubble/flow," in IUTAM conference on bubble dynamics and interfacial phenomena (ed. J. R. Blake), Kluwer, 1994.

[2] T.T. Bui, E.T. Ong, B.C. Khoo, E. Klaseboer, and K.C. Hung, "A fast algorithm for modeling multiple bubble dynamics," Journal of Computational Physics, vol. 216, pp. 430-453, 2006.

[3] $\mathrm{Z}$. Fu and V. Popov, "The adaptive cross approximation accelerated boundary element method for bubble dynamics," in Boundary Elements and Other Mesh Reduction Methods XXXIV, Eds. C. A. Brebbia and D. Poljak, Transactions of the Wessex Institute, Modelling and Simulation, vol. 53, 209-219, 2012.

[4] C.A. Brebbia and J. Dominguez, Boundary elements an introductory course. Southampton, UK: Computational Mechanics Publications, 1989.

[5] M. Bebendorf, Hierarchical matrices. A means to efficiently solve elliptic boundary value problems. Berlin: Lecture Notes in Computational Science and Engineering, 63, Springer-Verlag, 2008.

[6] K.W. Commander and A. Prosperetti, "Linear pressure waves in bubbly liquids: Comparison between theory and experiments," J. Acoust. Soc. Am., vol. 85, no. 2, pp. 732-746, 1989. 